\title{
Three graviton scattering and recoil effects in Matrix theory
}

\author{
A. Refolli ${ }^{a}$, N. Terzi ${ }^{b}$ and D. Zanon ${ }^{b}$ \\ ${ }^{a}$ Instituut voor Theoretische Fysica, Katholieke Universiteit Leuven \\ Celestijnenlaan 200D B-3001 Leuven, Belgium \\ E-mail: 'andrea.refolli@fys.kuleuven. ac.be' \\ ${ }^{b}$ Dipartimento di Fisica dell'Università di Milano and \\ INFN, Sezione di Milano, Via Celoria 16,20133 Milano, Italy \\ E-mail: 'terzi@pcteor1.mi.infn.it', E-mail: daniela.zanon@mi.infn.it',
}

Abstract: We discuss recent results on three gravitons in $M$-atrix theory at finite $N$. With a specific choice of the background we obtain the complete result up to two loops. The contributions from three-body forces agree with the ones presented in recent papers. We evaluate the two-body exchanges as well. We show that the result we have obtained from $M$-atrix theory precisely matches the result from one-particle reducible tree diagrams in eleven-dimensional supergravity .

$\mathrm{M}$ -THEORY seems to be a consistent quantum theory which includes eleven dimensional supergravity as a low-energy approximation. Since the introduction of the matrix model of $M$-theory [i] much progress has been made on the subject. According to the original conjecture, the degrees of freedom of $M$-theory in the infinite momentum frame are contained in the dynamics of $N D$ 0-branes in the $N \rightarrow \infty$ limit. Subsequently it was argued that $M$-theory with one of the lightlike coordinate compactified is in fact equivalent to the super Yang-Mills matrix

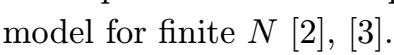

A crucial test of the conjecture consists in the comparison of graviton scattering in supergravity with corresponding results from $\mathrm{M}$ (atrix) theory in the low energy limit. So far compelling tests of this proposal have been the comparison of two-

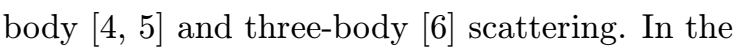
latter case there have been also some partial re-

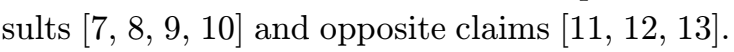
We focus on the $M$-atrix theory at finite $\bar{N}$ and explore further its correspondence with eleven dimensional supergravity. In particular we consider $N=3$ and compute graphs up to two-loops in $(0+1)$-dimensional Yang-Mills [1] ${ }^{1}$ - 1 . We find that they perfectly reproduce the three graviton scattering in supergravity, both in the direct three-body channel and in the two-body recoil exchange. The former, which corresponds to one-particle irreducible diagrams in supergravity, has been computed in the first paper of ref. [6] and we confirm that result. We complete the two-loop effective action calculation and evaluate two-body exchanges as well. We have found a systematic way to separate the light and heavy matrix model degrees of freedom which allows to obtain the full answer. We show that these contributions exactly match what expected from the two-loop scattering of two $D 0$-branes in $M$-atrix

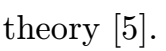

In the two body process there are only two expansion parameters: the relative velocity $v$ and the inverse of the relative spatial separation $r$. In the low energy limit, from a supergravity compu-

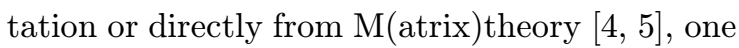
obtains the following effective Lagrangian

$$
L=\frac{v^{2}}{2 R}+\frac{15}{16 R^{3} M^{9}} \frac{v^{4}}{r^{7}}+\frac{225}{64 R^{5} M^{18}} \frac{v^{6}}{r^{14}}+O\left(\frac{v^{8}}{r^{21}}\right)
$$




\section{Three graviton scattering in M(atrix) theory}

In the following we will describe the full two loops effective potential calculation for the case of a three body scattering as computed in Matrix theory.

Our starting point is $M$-atrix theory at finite $N$ and we are interested in calculating the twoloop effective action and to compare it to that coming from eleven dimensional supergravity.

We will consider super Yang Mills theory reduced to $(0+1)$ dimensions with gauge group $U(3)$. We will use a formalism that easily allows to distinguish between the contributions from a direct three-body channel and the ones corresponding to a two-body recoil exchange.

The idea developed by [i6] is that true threebody contributions are those which depend on all the three relative velocities of the gravitons; all the rest should sum up to give a two-body scattering. The calculation we present shows in detail how this happens.

In the next section we give the explicit form of the gauged fixed action. The various fields are decomposed in terms of components on a $U(3)$ basis of hermitian matrices. The classical background is fixed with the three $D$-particles having relative velocities parallel to each others, orthogonal to the corresponding relative displacements. The result is analyzed keeping separate the two types of contributions mentioned above, i.e. terms which depend on two distinct relative velocities (three-body interaction) and terms in which only one relative velocity appears (recoil). Part of the calculations have been performed with the help of Mathematica.

\section{The action}

The matrix model is simply obtained by reducing $(9+1)$-dimensional $U(N)$ super Yang-Mills [1] $\left.{ }_{1}^{6}\right]$ to $(0+1)$ dimensions. This theory describes a system of $N D$-branes [1 17 in terms of nine bosonic fields $X_{i}$ and of sixteen fermionic superpartners $\theta$, which are spinors under $S O(9)$. The Euclidean action is given by

$$
S=\operatorname{Tr} \int d \tau\left\{\left(D_{\tau} X_{i}\right)^{2}-\frac{g}{2}\left[X_{k}, X_{j}\right]\left[X_{k}, X_{j}\right]\right.
$$

$$
\left.+\theta^{T} D_{\tau} \theta-\sqrt{g} \theta^{T} \gamma^{k}\left[X_{k}, \theta\right]\right\}
$$

where we have denoted by $g$ the Yang-Mills coupling constant and by $\gamma^{i}$ nine real, symmetric gamma matrices satisfying $\left\{\gamma^{i}, \gamma^{j}\right\}=2 \delta^{i j}$. The covariant derivative is defined by

$$
D_{\tau}=\partial_{\tau}-i \sqrt{g}[A
$$

The fields $X_{i}, \theta$ and $A$ are $N \times N$ hermitian matrices of $U(N)$, with $i, j, k=1,2, \ldots, 9$.

Being interested in quantum, perturbative calculations it is convenient to use the background field method, which allows to maintain explicit the gauge invariance of the result. To this end one expands the action (12.1) around a classical background field configuration $B_{i}$, setting $X_{i} \rightarrow$ $X_{i}+B_{i}$.

After gauge fixing and background splitting one finds the complete action:

$$
\begin{aligned}
S=\operatorname{Tr} & \int d \tau\left\{\left(\partial_{\tau} X_{i}\right)^{2}-\left[B_{k}, X_{j}\right]^{2}\right. \\
& -2 \sqrt{g}\left[B_{k}, X_{j}\right]\left[X_{k}, X_{j}\right]-\frac{g}{2}\left[X_{k}, X_{j}\right]\left[X_{k}, X_{j}\right] \\
& +\partial_{\tau} A^{2}-\left[A, B_{k}\right]^{2}-4 i \partial_{\tau} B_{k}\left[A, X_{k}\right]- \\
& 2 i \sqrt{g} \partial_{\tau} X_{k}\left[A, X_{k}\right]+2 \sqrt{g}\left[A, B_{k}\right]\left[X_{k}, A\right] \\
& -g\left[A, X_{k}\right]^{2}+\theta^{T} \partial_{\tau} \theta-i \sqrt{g} \theta^{T}[A, \theta] \\
& -\sqrt{g} \theta^{T} \gamma^{k}\left[X_{k}, \theta\right]-\theta^{T} \gamma^{k}\left[B_{k}, \theta\right] \\
& -2 \tilde{G} \partial_{\tau}^{2} G-2 i \sqrt{g} \partial_{\tau} \tilde{G}[A, G] \\
& \left.+2 \tilde{G}\left[B_{k},\left[B_{k}, G\right]-\sqrt{g}\left[G, X_{k}\right]\right]\right\}
\end{aligned}
$$

Here $X_{i}, A$ and $\theta$ are the quantum fluctuations, $G$ and $\tilde{G}$ are the ghosts, while $B_{k}$ is the external background.

We want to extract results to be compared to the scattering of three gravitons in supergravity, thus the minimal choice for the Yang-Mills gauge group that allows to describe the interaction of three $D 0$-branes, is $U(3)$. In fact the free motion of center of mass can be factored out; we thus deal only with a $S U(3)$ gauge group.

\subsection{Cartan Lie algebra for $S U(3)$}

In order to clearly distinguish between light and heavy Matrix model degrees of freedom it is convenient to use the Cartan basis for the Lie algebra of $S U(3)$. With such a basis every matrix field is 
decomposed into components as

$$
\begin{aligned}
X_{k} & \equiv X_{k}^{a} H_{a}+X_{k}^{\alpha} E_{\alpha}+X_{k}^{* \alpha} E_{-\alpha} \\
A & \equiv A^{a} H_{a}+A^{\alpha} E_{\alpha}+A^{* \alpha} E_{-\alpha} \\
\theta & \equiv \theta^{a} H_{a}+\theta^{\alpha} E_{\alpha}+\theta^{* \alpha} E_{-\alpha} \\
G & \equiv G^{a} H_{a}+G^{\alpha} E_{\alpha}+G^{* \alpha} E_{-\alpha} \\
\tilde{G} & \equiv \tilde{G}^{a} H_{a}+\tilde{G}^{* \alpha} E_{\alpha}+\tilde{G}^{\alpha} E_{-\alpha}
\end{aligned}
$$

where $H$ are the generator of Cartan subalgebra, $E$ are the Cartan step operators and $\alpha=$ $\pm \alpha^{1}, \pm \alpha^{2}, \pm \alpha^{3}$.

\subsubsection{Background choice}

Now we make a specific choice of the background configuration, i.e. straight line trajectories for the three particles. This amounts to have $B_{k}$ in diagonal form with

$$
B_{k}^{\alpha}=\tilde{v}_{k}^{\alpha} \tau+\tilde{b}_{k}^{\alpha} \quad \alpha=1,2,3
$$

As mentioned above the free motion of the center of mass can be factored out and ignored imposing

$$
\sum_{\alpha=1}^{3} \tilde{v}_{k}^{\alpha}=0 \quad \sum_{\alpha=1}^{3} \tilde{b}_{k}^{\alpha}=0
$$

We simplify further our calculations considering the case of parallel velocities for all three particles, e.g. along the $x_{1}$ axis, and relative displacements transverse [i]

$$
\begin{array}{ll}
\tilde{v}_{1}^{\alpha} \neq 0 \quad, \quad \tilde{v}_{k}^{\alpha}=0 \quad \text { for } \quad k>1 \\
\sum_{k=1}^{9} \tilde{v}_{k}^{\alpha} \tilde{b}_{k}^{\alpha}=0 & \text { for } \quad \alpha=1,2,3
\end{array}
$$

Setting $\tilde{v}_{1}^{\alpha}=\tilde{v}_{\alpha}$ the background matrices become

$B_{1}=\left(\begin{array}{ccc}\tilde{v}_{1} \tau & 0 & 0 \\ 0 & \tilde{v}_{2} \tau & 0 \\ 0 & 0 & \tilde{v}_{3} \tau\end{array}\right), \quad B_{k}=\left(\begin{array}{ccc}\tilde{b}_{k}^{1} & 0 & 0 \\ 0 & \tilde{b}_{k}^{2} & 0 \\ 0 & 0 & \tilde{b}_{k}^{3}\end{array}\right)$

for $k>1$.

We define relative velocities

$$
v_{1}=\tilde{v}_{2}-\tilde{v}_{3} \quad \text { and } \quad \text { cyclic }
$$

and relative impact parameters

$$
b_{k}^{1}=\tilde{b}_{k}^{2}-\tilde{b}_{k}^{3} \quad \text { and } \quad \text { cyclic }
$$

In terms of these quantities, setting

$$
R_{k}^{\alpha}=\sum_{a=1,2} \alpha_{a} \operatorname{Tr}\left(H^{a} B_{k}\right)
$$

one obtains

$$
R_{k}^{\alpha}=\left\{\begin{array}{ccc}
v_{\alpha} \tau & \text { if } & k=1 \\
b_{k}^{\alpha} & \text { if } & k>1
\end{array}\right.
$$

Now we can go back to the action in (2.3i) and perform the trace operation explicitly, using the notation

$$
\begin{aligned}
\left(b^{\alpha}\right)^{2} & \equiv \sum_{k} b_{k}^{\alpha} b_{k}^{\alpha} \\
\left(R^{\alpha}\right)^{2} & \equiv \sum_{k} R_{k}^{\alpha} R_{k}^{\alpha} \equiv v_{\alpha}^{2} \tau^{2}+\left(b^{\alpha}\right)^{2}
\end{aligned}
$$

For example the terms involving the $X$ fields are found to be:

$$
\begin{aligned}
S_{X}= & \int d \tau\left\{X_{k}^{a}\left(-\partial_{\tau}^{2}\right) X_{k}^{a}+2 X_{k}^{* \alpha}\left(-\partial_{\tau}^{2}+\left(R^{\alpha}\right)^{2}\right) X_{k}^{\alpha}\right. \\
& -2 \sqrt{g}\left[\epsilon ^ { \alpha \beta \gamma } R _ { k } ^ { \gamma } \left(X_{k}^{\alpha} X_{j}^{\beta} X_{j}^{\gamma}\right.\right. \\
& \left.+X_{k}^{* \alpha} X_{j}^{* \beta} X_{j}^{* \gamma}\right)-2 R_{k}^{\alpha} X_{j}^{\alpha} X_{j}^{* \alpha} X_{k}^{a} \alpha_{a} \\
& \left.+R_{k}^{\alpha} X_{j}^{\alpha} X_{k}^{* \alpha} X_{j}^{a} \alpha_{a}+R_{k}^{\alpha} X_{k}^{\alpha} X_{j}^{* \alpha} X_{j}^{a} \alpha_{a}\right] \\
& -g\left[-2 X_{j}^{\alpha} X_{j}^{* \alpha}\left(X_{k}^{a} \alpha_{a}\right)^{2}-2 X_{k}^{a} \beta_{a} X_{j}^{\beta} X_{k}^{\alpha} X_{j}^{\gamma} \epsilon^{\alpha \beta \gamma}\right. \\
& +2 X_{k}^{a} \beta_{a} X_{j}^{b} \beta_{b} X_{j}^{\beta} X_{k}^{* \beta}-2 X_{k}^{a} \beta_{a} X_{j}^{* \beta} X_{k}^{* \alpha} X_{j}^{* \gamma} \epsilon^{\alpha \beta \gamma} \\
& -X_{k}^{\alpha} X_{k}^{* \eta} X_{j}^{\beta} X_{j}^{* \rho} \epsilon^{\alpha \beta \gamma} \epsilon^{\eta \rho \gamma}+X_{k}^{\alpha} X_{j}^{* \alpha} X_{k}^{\beta} X_{j}^{* \beta}(\alpha \cdot \beta) \\
& \left.\left.-X_{k}^{\alpha} X_{j}^{* \alpha} X_{j}^{\beta} X_{k}^{* \beta}(\alpha \cdot \beta)\right]\right\}
\end{aligned}
$$

¿From the quadratic part of the action one can easily read the mass matrix and obtain the following particle content in the spectrum:

- 8 complex bosons with mass $\left(R^{\alpha}\right)^{2}$ where $\alpha=1,2,3$;

- 1 complex boson with mass $\left(R^{\alpha}\right)^{2} \pm 2 v_{\alpha}$ where $\alpha=1,2,3$;

- 20 real, massless bosons;

- 2 complex ghost fields with mass $\left(R^{\alpha}\right)^{2}$ where $\alpha=1,2,3$;

- 2 real, massless ghosts;

- 1 complex spinor with mass $\gamma^{i} R_{i}^{\alpha}$ where $\alpha=1,2,3$;

- 2 Majorana, massless spinors.

According to the conjecture strings stretching between D0-branes are associated to massive degrees of freedom.

Correspondingly one obtains the propagators of the various fields [i4]. 


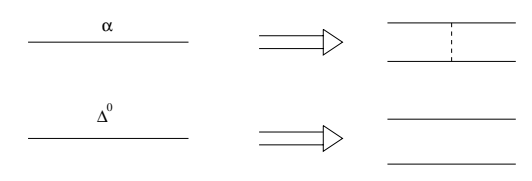

Figure 1: Correspondence between M(atrix) d.o.f. and D-particle interactions by

For example bosonic propagators are given

$$
\begin{aligned}
<X_{i}^{a}(\tau) X_{j}^{b}\left(\tau^{\prime}\right)> & =\frac{1}{2} \delta^{a b} \delta_{i j} \Delta_{0}\left(\tau, \tau^{\prime}\right) \\
<X_{i}^{* \alpha}(\tau) X_{j}^{\beta}\left(\tau^{\prime}\right)> & =\frac{1}{2} \delta^{\alpha \beta} \delta_{i j} \int_{0}^{\infty} \mathrm{d} s \Delta^{\alpha}\left(\tau, \tau^{\prime}, s\right) \\
i, j \neq 1 &
\end{aligned}
$$

We have defined

$$
\Delta_{0}\left(\tau, \tau^{\prime}\right)=\theta\left(\tau-\tau^{\prime}\right)\left(\tau^{\prime}-\tau\right)
$$

and

$$
\begin{aligned}
\Delta^{\alpha}\left(\tau, \tau^{\prime}, s\right)= & e^{-\left(b^{\alpha}\right)^{2} s} \sqrt{\frac{v_{\alpha}}{2 \pi \operatorname{Sinh}\left(2 s v_{\alpha}\right)}} \\
& e^{-v_{\alpha} T^{2} \operatorname{Tanh}\left(s v_{\alpha}\right)-v_{\alpha} t^{2} \operatorname{Coth}\left(s v_{\alpha}\right)}
\end{aligned}
$$

where we have introduced new time variables

$$
T=\frac{1}{2}\left(\tau+\tau^{\prime}\right) \quad t=\frac{1}{2}\left(\tau-\tau^{\prime}\right)
$$

In the fermionic sector the propagators are given by

$$
\begin{gathered}
<\theta^{a T}(\tau) \theta^{b}\left(\tau^{\prime}\right)>=\frac{1}{2} \delta^{a b} \partial_{\tau} \Delta_{0}\left(\tau, \tau^{\prime}\right) \\
<\theta^{* \alpha T}(\tau) \theta^{\beta}\left(\tau^{\prime}\right)>=\frac{1}{2} \delta^{\alpha \beta} \Delta_{\mathbf{F}}^{\alpha}\left(\tau, \tau^{\prime}\right)
\end{gathered}
$$

We have defined

$$
\begin{aligned}
& \Delta_{0}\left(\tau, \tau^{\prime}\right)=\theta\left(\tau-\tau^{\prime}\right)\left(\tau^{\prime}-\tau\right) \\
& \boldsymbol{\Delta}_{\mathbf{F}}^{\alpha}\left(\tau, \tau^{\prime}\right) \equiv \int_{0}^{\infty} \mathrm{d} s\left(-\mathbb{I} \frac{v_{\alpha} t}{\operatorname{Sinh}\left(v_{\alpha} s\right)}+\gamma^{1} \frac{v_{\alpha} T}{\operatorname{Cosh}\left(v_{\alpha} s\right)}\right. \\
& \left.\quad+\phi^{\alpha} \operatorname{Cosh}\left(v_{\alpha} s\right)+\not \phi^{\alpha} \gamma^{1} \operatorname{Sinh}\left(v_{\alpha} s\right)\right) \Delta^{\alpha}\left(\tau, \tau^{\prime}, s\right)
\end{aligned}
$$

Once the propagators are known the oneloop contribution to the effective action is easily

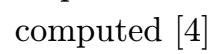

$$
\Gamma^{(1)}=-\frac{15}{16} \sum_{\alpha=1}^{3} \int d T \frac{\left(v_{\alpha}\right)^{4}}{\left[\left(b^{\alpha}\right)^{2}+T^{2}\left(v_{\alpha}\right)^{2}\right]^{\frac{7}{2}}}
$$

In the next section we will present the twoloop calculation.

\section{The two-loop effective action}

The two-loop contributions are evaluated considering two types of diagrams, the ones involving one four-point vertex, the figure-eight graphs, and the ones with two three-point vertices, the sunset-type graphs, respectively. In the present notation they correspond to (Fig 2).
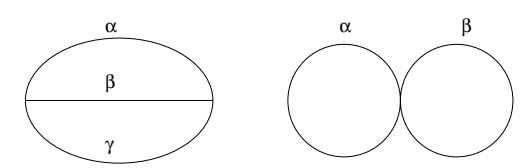

Figure 2: Typical diagrams: the Greek indices correspond to massive degrees of freedom

Given this setup, one can distinguish between the graphs that lead to genuine three-body exchanges (one-particle irreducible diagrams for the three-graviton scattering in supergravity) and twobody recoil effects (one-particle reducible graphs in supergravity) in a very simple manner. We need collect terms that depend on two independent relative velocities on one side, and terms that depend only on one relative velocity on the other side.

Thus from the figure-eight diagrams one has a three-body exchange whenever the two propagators come in with different masses, while a recoil term is produced when the two masses are equal. The connection between these graphs and supergravity diagrams is shown in (Fig 3). For example in the first diagram, since the graph depends on a single $E_{\alpha}$, we have a string (graviton) stretching between only two D-particles. As we have seen this corresponds to a string (fluctuation).

The presence of a massless propagator would lead to a vanishing contribution for this tadpole kind of diagrams.

¿From the sunset-type graphs we have direct three-body contributions when the three propagators have three distinct masses (three different relative velocities out of which two are independent). Two-body forces are present when two masses are equal, being the third one equal 


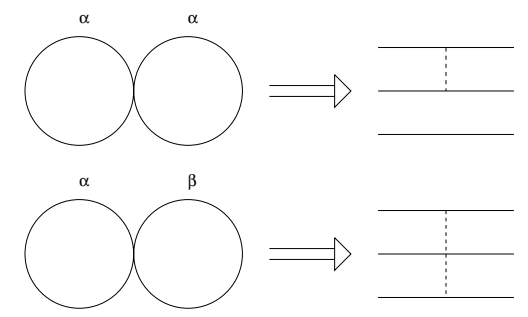

Figure 3: Correspondence between M(atrix) graphs and supergravity tree diagrams

to zero as required by momentum conservation. Having decomposed the fields as in (2.) makes it easy to identify the massless particles which are given simply by the diagonal degrees of freedom.

Now we present the results obtained using this procedure [1] contributions to the three-body forces ${ }^{1}$ Then we will concentrate on the two-loop contributions that depend only on one relative velocity $v_{\alpha}$. Their complete evaluation is conceptually simple and algebraically manageable within our approach. In [1 14 in we have isolated the leading order term and proved its consistency with the re-

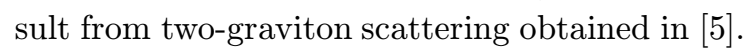
This shows without any further ambiguity that we are dealing with recoil effects.

\subsection{Three-body contributions}

As emphasized above one needs to compute all the two-loop terms which depend on two distinct relative velocities ${ }^{2}$, and this implies that only propagators associated to off-diagonal degrees of freedom (see $(2 .-\overline{4}))$ will enter this part of the calculation.

In [114] we have presented the details of the calculation, here we summarize the result. The final answer can be rearranged as a sum of two contributions

$$
\Gamma=\Gamma_{V}+\Gamma_{Y}
$$

Explicit calculations lead to

$\Gamma_{V}=-\sum_{\beta \neq \gamma} \int d T d s_{2} d s_{3} 128 \operatorname{Sinh}^{3}\left(\frac{s_{2} v_{\beta}}{2}\right) \operatorname{Sinh}^{3}\left(\frac{s_{3} v_{\gamma}}{2}\right)$

\footnotetext{
${ }^{1} \mathrm{We}$ will follow as much as possible the approach in ref. [6] so that a direct comparison can be made in a straightforward manner. Complete agreement with their findings is shown in the next subsection.

${ }^{2}$ In sunrise diagrams there is a third relative velocity which is fixed due to momentum conservation
}

$$
\begin{aligned}
\times & \left(2 \operatorname{Cosh}\left(\frac{s_{2} v_{\beta}}{2}\right) \operatorname{Cosh}\left(\frac{s_{3} v_{\gamma}}{2}\right)-\right. \\
& \left.\operatorname{Sinh}\left(\frac{s_{2} v_{\beta}}{2}\right) \operatorname{Sinh}\left(\frac{s_{3} v_{\gamma}}{2}\right)\right) \Delta^{\beta}\left(s_{2}\right) \Delta^{\gamma}\left(s_{3}\right)_{\left.\right|_{t=0}}
\end{aligned}
$$

and expanding at the leading order

$$
\begin{aligned}
\Gamma_{Y}= & -\sum_{\alpha \neq \beta \neq \gamma} \int d T d t d s_{1} d s_{2} d s_{3} \frac{1}{18}\left(s_{1} v_{\alpha}-s_{2} v_{\beta}\right)^{2} \\
& \left(s_{1} v_{\alpha}-s_{3} v_{\gamma}\right)^{2}\left(s_{2} v_{\beta}-s_{3} v_{\gamma}\right)^{2} \\
& \left(s_{1} v_{\alpha}^{2}+s_{2} v_{\beta}^{2}+s_{3} v_{\gamma}^{2}\right) \Delta^{\alpha}\left(s_{1}\right) \Delta^{\beta}\left(s_{2}\right) \Delta^{\gamma}\left(s_{3}\right)
\end{aligned}
$$

(13.1יi) exactly reproduces the corresponding term obtained in ref. [i]

Now we turn to the calculation of the recoil effects.

\subsection{Two-body recoil contributions}

We are considering here all the diagrams not computed in the previous section, i.e. figure-eight graphs with the two propagators carrying the same mass, and sunset-type graphs with two propagators of equal masses and the third one massless.
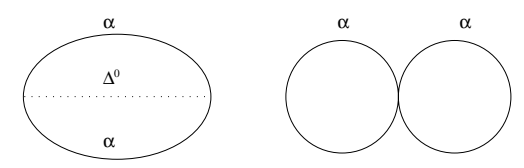

Figure 4: All the remaining graphs

Looking at the spectrum of the various particles it is clear that these contributions depend on a single relative velocity $v_{\alpha}$ and are thus candidates to represent supergravity two-body recoil effects. In [1 1 , ] we have determined them exactly and shown that the above interpretation is indeed correct. We find

$$
\begin{aligned}
& \Gamma_{\text {recoil }}= \\
& \quad-\sum_{\alpha}\left[\int d s_{1} d s_{2} \frac{\left(\left(b^{\alpha}\right)^{2} V_{1}+V_{2}\right) e^{-\left(b^{\alpha}\right)^{2}\left(s_{1}+s_{2}\right)}}{2 \pi v_{\alpha} \sqrt{s_{1} s_{2}} \sqrt{s_{1}+s_{2}}}\right]
\end{aligned}
$$

where (up to order $v_{\alpha}^{6}$ ) we find

$$
\begin{aligned}
V_{1} & =\left(75600+22680 s_{1}^{2} v_{\alpha}^{2}+2520 s_{1} s_{2} v_{\alpha}^{2}\right. \\
& +22680 s_{2}^{2} v_{\alpha}^{2}+630 s_{1}^{4} v_{\alpha}^{4}-2100 s_{1}^{3} s_{2} v_{\alpha}^{4} \\
& +10290 s_{1}^{2} s_{2}^{2} v_{\alpha}^{4}-2100 s_{1} s_{2}^{3} v_{\alpha}^{4}+630 s_{2}^{4} v_{\alpha}^{4} \\
& +139 s_{1}^{6} v_{\alpha}^{6}+761 s_{1}^{5} s_{2} v_{\alpha}^{6}+1794 s_{1}^{4} s_{2}^{2} v_{\alpha}^{6} \\
& -947 s_{1}^{3} s_{2}^{3} v_{\alpha}^{6}+1794 s_{1}^{2} s_{2}^{4} v_{\alpha}^{6}+761 s_{1} s_{2}^{5} v_{\alpha}^{6} \\
& \left.+139 s_{2}^{6} v_{\alpha}^{6}\right) \frac{s_{1} s_{2}}{840\left(s_{1}+s_{2}\right)}
\end{aligned}
$$




$$
\begin{aligned}
V_{2}= & \left(-75600 s_{1}^{2}+302400 s_{1} s_{2}-75600 s_{2}^{2}\right. \\
& -17640 s_{1}^{4} v_{\alpha}^{2}-27720 s_{1}^{3} s_{2} v_{\alpha}^{2}-277200 s_{1}^{2} s_{2}^{2} v_{\alpha}^{2} \\
& -27720 s_{1} s_{2}^{3} v_{\alpha}^{2}-17640 s_{2}^{4} v_{\alpha}^{2}-6510 s_{1}^{6} v_{\alpha}^{4} \\
& +2940 s_{1}^{5} s_{2} v_{\alpha}^{4}+68040 s_{1}^{4} s_{2}^{2} v_{\alpha}^{4} \\
& +211680 s_{1}^{3} s_{2}^{3} v_{\alpha}^{4}+68040 s_{1}^{2} s_{2}^{4} v_{\alpha}^{4} \\
& +2940 s_{1} s_{2}^{5} v_{\alpha}^{4}-6510 s_{2}^{6} v_{\alpha}^{4}-97 s_{1}^{8} v_{\alpha}^{6} \\
& +2065 s_{1}^{7} s_{2} v_{\alpha}^{6}-7695 s_{1}^{6} s_{2}^{2} v_{\alpha}^{6}-51738 s_{1}^{5} s_{2}^{3} v_{\alpha}^{6} \\
& -103508 s_{1}^{4} s_{2}^{4} v_{\alpha}^{6}-51738 s_{1}^{3} s_{2}^{5} v_{\alpha}^{6}-7695 s_{1}^{2} s_{2}^{6} v_{\alpha}^{6} \\
& \left.+2065 s_{1} s_{2}^{7} v_{\alpha}^{6}-97 s_{2}^{8} v_{\alpha}^{6}\right) \frac{1}{3360\left(s_{1}+s_{2}\right)^{2}}
\end{aligned}
$$

Finally the integrations on $s_{1}$ and $s_{2}$ can be performed and summing the two contributions one obtains

$$
\Gamma_{\text {recoil }}=-\sum_{\alpha} \frac{51975}{65536} \pi \frac{v_{\alpha}^{5}}{\left(b^{\alpha}\right)^{13}}
$$

If we write (3.2) in the form

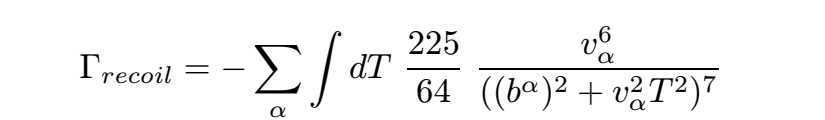

then this expression can be directly compared with the result in (i) where the two graviton scattering was analyzed: we find that numbers perfectly match. The expression in (3.3i) with $\Gamma_{V}$ and $\Gamma_{Y}$ determines the full two-loop contribution to the three D-particle effective action.

\section{Conclusions}

In this talk we have presented some checks of the finite $N \mathrm{M}$ (atrix) theory conjecture [2in]. These checks consist in comparing the $\mathrm{M}$ (atrix) theory quantum low energy effective action in a scattering process between gravitons with analogous amplitudes from eleven dimensional supergravity compactified on a lightlike circle.

In particular we considered a three particle process for which it has been determined the full two-loop calculation in $M$-atrix theory.

Following [1] 1 ind _we have restricted our attention

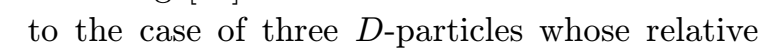
velocities are parallel and orthogonal to the corresponding impact parameters. This restriction has been forced on us by the necessity of keeping calculations mageable. First we have found that this calculation confirms what obtained in $[6]$ showing that the predictions from one-particle irreducible tree diagrams in supergravity are in agreement with two-loop contributions from $M$ atrix theory.

In addition we have considered those two-loop contributions which depend only on one relative velocity between the gravitons. We have shown how these terms give a total sum which is consistent with results from a two-loop calculation of two $D$-particle scattering [ $[\overline{5}]]$. This calculation gives additional support to the idea that eleven-dimensional supergravity compactified in a null direction is in direct correspondence with $M$-atrix theory at finite $N$.

\section{References}

[1] T. Banks, W Fischler, S.H. Shenker, L. Susskind, Phys. Rev. D $\mathbf{5 5}(1997)$; 5112. [hep-th/9610043]

[2] Leonard Susskind Another Conjecture about $M$ (atrix) Theory hep-th/9704080'

[3] N. Seiberg, Why is the Matrix model correct? IPhys. Rev. Lett. $\mathbf{7 9}(1997) !$ 3577 ihep-th/9710009]

[4] K. Becker, M. Becker, A Two-Loop Test of $M$ (atrix) Theory iNucl. Phys. B 506 (1997) 48' [hep-th/9705091]

[5] K. Becker, M. Becker, J. Polchinski, A. Tseytlin, Higher Order Graviton Scattering in M(atrix) Theory 'Phys. Rev. D $\mathbf{5 6}(1997)$ 3174' hep-th/9706072i

[6] Y. Okawa, T. Yoneya, Multi-Body Interactions of D-Particles in Supergravity and Matrix TheoryiNucl. Phys. B $\mathbf{5 3 8}(1999)$ ! $6 \overline{7}, 67$ [hep-th/9806108]; Equations of Motion and Galilei Invariance in D-Particle

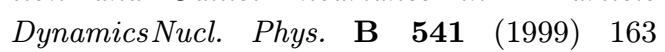
hep-th/9808188i]

[7] M. Fabbrichesi, G. Ferretti, R. Iengo, Supergravity and matrix theory do not disagree on multi-graviton scattering J. High Energy Phys.' 06 (1998) 27 [hep-th/9806018]

[8] W. Taylor, M. Raamsdonk, Three-graviton scattering in Matrix theory revisited Phys. Lett! B 438 (1998) 248 [hep-th/9806066!

[9] R. Echols, J. Grey, Comment on Multigraviton Scattering in the Matrix Model 'Phys. Lett. B' 449 (1999) 60 [hep-th/9806109] 
[10] J. McCarthy, L. Susskind, A. Wilkins, Large $N$ and the Dine-Rajaraman problem 'Phys. Lett.'

[ - - _ B 437 (1998) 62 [hep-th/9806136]

[11] M. Dine, A. Rajaraman, Multigraviton Scattering in the Matrix Model 'Phys. Lett. B 425!

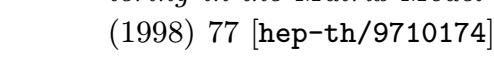

[12] Michael Dine, Robert Echols, Joshua P. Gray Tree Level Supergravity and the Matrix Model hep-th/9810021'

[13] R. Helling, J. Plefka, M. Serone, A. Waldron Three Graviton Scattering in M-Theory 'Nucl.' ; - - - Phys. B $\mathbf{5} 59$ (1999) 184 [hep-th/9905183]

[14] A. Refolli, N. Terzi, D. Zanon Threegraviton scattering and recoil effects in $M$ atrix theory 'Nucl. Phys. B $\mathbf{5 6 4}(2000)$ 241' hep-th/9905091"

[15] Iliopoulos, Itzykson and Martin Functional Methods and Perturbation Theory'Rev. Mod.' '- - - - Phys. 46 (1975) 165!

[16] M. Claudson, M. Halpern, Supersymmetric ground state wave functions Nucl. Phys. B.520 (1985) $689^{\prime}$

R. Flume, On quantum mechanics with extended supersymmetry and nonabelian gauge contraints Ann. Phys. $(N Y) \mathbf{1} \mathbf{6} 4(1985)$ 189 M. Baake, P. Reinecke, V. Rittenberg, Fierz identities for real Clifford algebras and the number of supercharges iJ. Math. Phys. $\mathbf{2}_{1}$ - - $(1985)$ - 1070

[17] J. Polchinski, Dirichlet-Branes and RamondRamond Charges iPhys. Rev. Lett. $\mathbf{7 5}(1995)$

'- - - - - 4724 '

E. Witten, Bound States Of Strings And p-Branes 'Nucl. Phys. B 460 (1996) 335! hep-th/9510135 